\title{
Análisis de diversidad de la familia Poaceae en la región austral de America del Sur
}

\author{
Analysis of Poaceae biodiversity in austral South America
}

Fernando Biganzoli ${ }^{1} \&$ Fernando Zuloaga $^{2,3}$

\begin{abstract}
Resumen
Análisis de diversidad de la familia Poaceae en la región austral de América del Sur. La familia Poaceae es una de las mejor representadas en América del Sur austral, con un total de 206 géneros que comprenden 1523 especies distribuidas en 10 subfamilias diferentes. En este trabajo analizamos la distribución de estos grupos en Argentina, sur de Brasil (Paraná, Rio Grande do Sul y Santa Catarina), Chile, Paraguay y Uruguay; discutimos la riqueza de las diferentes subfamilias, tribus y géneros, su distribución, especies endémicas, la relación de especies anuales y perennes, así como el número de especies Kranz y no Kranz, su abundancia según regiones geográficas, en relación con la temperatura y las precipitaciones, géneros disyuntos, y la proporción de taxones en relación con las diferentes.

Palabras-claves: diversidad, $\mathrm{C}_{3}, \mathrm{C}_{4}$, especies introducidas, especies anuales y perennes, provincias biogeográficas.

Abstract

Analysis of Poaceae biodiversity in austral South America. The Poaceae is one of the best represented families in austral South America with a total of 206 genera comprising 1523 species arranged in 10 different subfamilies. Here we analyzed the distribution of these taxa in Argentina, southern Brazil (Paraná, Rio Grande do Sul and Santa Catarina), Chile, Paraguay, and Uruguay; we discuss the species richness of the different subfamilies, tribes and genera, its distribution, endemics, annual and perennial species as well as Kranz and non Kranz taxa, its geographical distribution in relation to temperature and rainfall, disjunct genera, and proportion of taxa in relation to the different ecoregions in the area.
\end{abstract}

Key words: diversity, $\mathrm{C}_{3}, \mathrm{C}_{4}$, exotic species, annual and perennial species, biogeographic provinces.

\section{Introducción}

La familia Poaceae incluye aproximadamente 700 géneros y 11000 especies (Chen et al. 2006) $\mathrm{y}$ es una de las 4 familias con mayor número de especies de plantas vasculares. Esta familia se distribuye prácticamente sobre toda la superficie de la Tierra, desde los trópicos hasta los círculos polares; de las 2 especies de plantas vasculares nativas que crecen en la Antártida, una es gramínea: Deschampsia antarctica E. Desv. (Chiapella \& Zuloaga 2010). Muchos de los géneros y taxones de Poaceae son dominantes en una gran diversidad de ecosistemas. Sus especies están presentes en hábitats tan variados como bosques tropicales, sabanas, desiertos y semidesiertos, pampas, estepas, etc., y si bien no son particularmente abundantes en los bosques sí son dominantes en ambientes abiertos como sabanas, estepas y praderas.

Además de su amplia distribución, tanto en términos geográficos como ecológicos, las gramíneas poseen un enorme valor económico para el hombre; entre sus especies se encuentran algunos de los cultivos de mayor importancia, como el trigo (género Triticum L.), el arroz (Oryza L.), el maíz (Zea L.), la cebada (Hordeum L.), el centeno (Secale L.), las avenas (Avena L.), la caña de azúcar (Saccharum L.) y el tef (Eragrostis Wolf). En adición, numerosas especies se utilizan

\footnotetext{
${ }^{1}$ Departamento de Métodos Cuantitativos y Sistemas de Información, Facultad de Agronomía, Universidad de Buenos Aires, Buenos Aires, Argentina.

${ }^{2}$ Instituto de Botánica Darwinion, Labardén 200, Casilla de Correo 22, B1642HYD, San Isidro, Buenos Aires, Argentina.

${ }^{3}$ Autor para la correspondencia: fzuloaga@darwin.edu.ar
} 
como forrajeras, naturales o cultivadas, como por ejemplo especies del género Urochloa $\mathrm{P}$. Beauv.; en pasturas naturales de América del Sur, son importantes algunas especies de gramíneas nativas de los géneros Festuca L., Nassella (Trin.) Desv., Pappostipa (Speg.) Romasch., P.M. Peterson \& Soreng, Poa L., Bromus L., Paspalum L. y Hordeum. Otras especies son importantes ya que son fijadoras de dunas (Panicum L., Spartina Schreb.), o se utilizan como césped en parques, estadios y campos de golf (Lolium L., Stenotaphrum, Trin., Zoysia Willd.); otras son aromáticas y se utilizan en la producción de aceites esenciales aplicados en perfumería como la citronella y el vetiver (géneros Elionurus Humb. \& Bonpl. ex Willd. y Chrysopogon Trin.). Recientemente, algunas especies comenzaron a utilizarse con fines ornamentales, como por ejemplo especies de los géneros Cenchrus L., Cortaderia Stapf o Panicum. Asimismo, diversas especies son importantes malezas de cultivos, principalmente especies de los géneros Eleusine Gaertn., Cynodon Rich., Dactyloctenium Willd., o son tóxicas para el ganado, como algunas especies de los géneros Festuca y Nassella, entre otros.

La taxonomía de la familia se basó, en sus inicios, en la estructura de la espiguilla y las diferentes variantes observadas en las mismas y en la inflorescencia. Es así que Bentham \& Hooker (1880) dividieron a la familia en las series Panicaceae y Poaceae, basadas en el número de flores por espiguilla y la articulación de la misma; estos grupos fueron luego llamados subfamilias Panicoideae y Pooideae (Hitchcock 1951). A partir de 1930, la clasificación de la familia sufrió constantes cambios debido al análisis de nuevos caracteres anatómicos, citológicos, embriológicos, fisiológicos y biogeográficos, entre otros. Así, Pilger (1956) acepta un total de 8 subfamilias y Parodi (1961) seis: Bambusoideae, Oryzoideae, Phragmitoideae, Festucoideae, Eragrostoideae y Panicoideae; esta última clasificación fue utilizada, en el área de estudio, por numerosos agrostólogos de Argentina, Brasil, Chile y Uruguay. Clayton \& Renvoize (1986) aportan una importante contribución al conocimiento de la familia, considerando, en una clasificación intuitiva, 6 subfamilias a nivel mundial: Arundinoideae, Bambusoideae, Centothecoideae, Chloridoideae, Panicoideae y Pooideae. Casi simultáneamente, Watson \& Dallwitz (1992) elaboran un estudio de géneros, también a nivel mundial, siguiendo la clasificación de subfamilias propuesta por Clayton \& Renvoize (1986). Estudios filogenéticos llevados a cabo en los últimos 25 años, basados en el análisis de ADN del cloroplasto y nuclear, reconocen actualmente un total de 12 subfamilias (GPWG I 2001; Sánchez-Ken et al. 2007; SánchezKen \& Clark 2010; GPWG II 2012; Linder et al. 2010; Morrone et al. 2012; Peterson et al. 2010; Soreng et al. 2011). En el presente tratamiento se sigue Soreng et al. (2011), según la cual se encuentran en el área de estudio las siguientes subfamilias: Anomochlooideae, Aristidoideae, Arundinoideae, Bambusoideae, Chloridoideae, Danthonioideae, Ehrhartoideae, Panicoideae, Pharoideae y Pooideae.

En esta contribución realizamos una sinopsis actualizada de la diversidad de Poaceae para el sur de América del Sur, con énfasis en la distribución geográfica de las especies nativas y exóticas de la familia, de los tipos fotosintéticos y del ciclo de vida.

Base de datos utilizada y disponibilidad de los datos: Para realizar este trabajo se utilizó la base de datos Documenta Florae Australis (en adelante DFA), la que está disponible a través de la página web del Instituto de Botánica Darwinion: $(<\mathrm{http}: / /$ www.darwin.edu.ar $>)$. Esta base de datos provee de un inventario actualizado y completo de las Poaceae del Cono Sur; la información se mantiene al día, representando un recurso valioso para el conocimiento, manejo y conservación de los taxones que la integran. Cada género y especie incluye datos sobre su publicación original, sinonimia, referencias bibliográficas, presencia en cada país y distribución en los mismos, estatus, hábito y ciclo de vida. Se incluyen también ejemplares de referencia y notas u observaciones cuando corresponde.

En resumen, la información se presenta en el siguiente formato:

1) Nombres aceptados, confirmados a través del análisis de investigadores, de la bibliografía correspondiente y con su correspondiente ejemplar de herbario. Los autores de taxones se estandarizaron siguiendo a Brummitt \& Powell (1992). A la fecha, DFA contiene información sobre 1523 especies válidas de Poaceae (en el presente trabajo no consideramos a los taxones infraespecíficos).

2) Los sinónimos aparecen luego del nombre válido, en orden cronológico, incluyendo la 
bibliografía correspondiente. Se consideran basónimos y sinónimos citados o relevantes para el área de estudio. A la fecha, DFA contiene 4770 sinónimos.

3) Referencias bibliográficas: toda la bibliografía utilizada para la evaluación de taxones se lista por autor y año de publicación. Estas referencias generales son incluidas con el objeto de brindar acceso a información más completa de cada taxón.

4) Presencia y distribución en los países del área: se indica la presencia en Argentina, sur de Brasil, Chile, Paraguay y Uruguay y las divisiones políticas dentro de cada país.

5) Hábito y ciclo de vida: se indica si el taxón es herbáceo o leñoso (en el caso de Poaceae) y si es anual o perenne cuando se conoce.

6) Estatus: se identifica si el taxón es nativo, endémico o introducido.

7) Ejemplares examinados: se cita al menos un ejemplar por país; en total se incluyen en DFA (a septiembre de 2014) 52500 representantes de Poaceae.

\section{Área del proyecto}

El área que abarca el presente trabajo incluye el extremo sur de Sudamérica, los territorios de Argentina, el sur de Brasil (estados de Paraná, Santa Catarina y Rio Grande do Sul), Chile, Paraguay y Uruguay (Fig. 1a). Esta área, que se ha denominado "Cono Sur", se extiende al sur del paralelo $20^{\circ} \mathrm{S}$ desde el Océano Atlántico al Océano Pacífico, excepto por la República de Bolivia y los estados brasileños de Mato Grosso do Sul y São Paulo. Los límites latitudinales se corresponden con la extensión norte-sur de Chile continental $\left(17^{\circ} 30^{\prime}-56^{\circ} 30^{\prime} \mathrm{S}\right)$, y los longitudinales se encuentran aproximadamente entre los $48^{\circ}-75^{\circ} \mathrm{O}$. Este territorio cubre un total de $4.708 .617 \mathrm{~km}^{2}$, lo que representa aproximadamente el $26 \%$ de la superficie total de América del Sur.

Dentro de la Argentina se consideraron las Islas Malvinas y la Isla de los Estados, mientras que no se incluyó el continente antártico e islas adyacentes. Para Chile se analizó la presencia de la familia en las islas integradas a su dominio, las que incluyen, entre las más importantes, a las islas de Pascua, Sala y Gómez, Robinson Crusoe, Alejandro Selkirk y Santa Clara (estas tres últimas pertenecientes al Archipiélago Juan Fernández), San Ambrosio y San Félix (Islas Desventuradas). En las figuras donde se incluyen límites políticos, algunas subdivisiones fueron eliminadas para obtener unidades de área similar.

\section{Regiones biogeográficas}

En el área del Cono Sur las investigaciones sobre plantas vasculares y su distribución han utilizado, en líneas generales, la clasificación propuesta por Cabrera (1976) para la Argentina, o de Cabrera \& Willink (1973) al nivel de América del Sur. No obstante, estos sistemas han sido debatidos recientemente por diversos autores (Morrone 2001; Ribichich 2002). En este trabajo seguimos, con algunas modificaciones, el esquema propuesto por Josse et al. (2003) (Fig. 1b). Para ello agrupamos las ecorregiones en 13 unidades a las que identificamos con los nombres propuestos por Cabrera \& Willink (1973), aunque igualmente se han considerado los trabajos clásicos de fitogeografía para América Latina y otros especializados para cada región. En los análisis, la ecorregión correspondiente a la provincia chaqueña fue agrupada con la del espinal. Para facilitar la interpretación de las figuras, las provincias altoandina, puna y prepuna fueron agrupadas (Fig. 1b).

\section{Resultados y Discusión}

En la actualidad se registran para el Cono Sur 1523 especies de Poaceae aceptadas, distribuidas en 206 géneros, 25 tribus y 10 subfamilias, con un total de 541 especies endémicas (Apéndice Tab. 1, Tab. 2 y Tab. 3).

Sólo 37 de los 206 géneros presentes en el área están compartidos por los 5 países del área, mientras que 36 géneros son exclusivos de uno de los 5 países (18 Argentina, 8 Brasil, 5 Chile y 5 Paraguay; Apéndice Tab. 4). La mayor cantidad de géneros compartidos entre dos de los países se da entre Argentina y Brasil con 118, aunque de ellos sólo 2 son exclusivos de esos dos países, mientras que de los 100 géneros compartidos entre Argentina y Chile, 27 no están presentes en los otros tres países de la región. El único género compartido exclusivamente entre dos países no limítrofes (Chile y Uruguay ) es el género introducido Miscanthus Andersson.

En el área hay un total de 9 géneros endémicos, de acuerdo al siguiente detalle: Chloridoideae, Cynodonteae: Neobouteloua Gould (presente en la Argentina y Chile); Panicoideae, Paspaleae: Oplismenopsis Parodi (Argentina y Uruguay) y Osvaldoa J.R. Grande (Argentina, sur de Brasil y Uruguay); Pooideae, Poeae: Leptophyllochloa C.E. Calderón (Argentina y Chile), Megalachne 


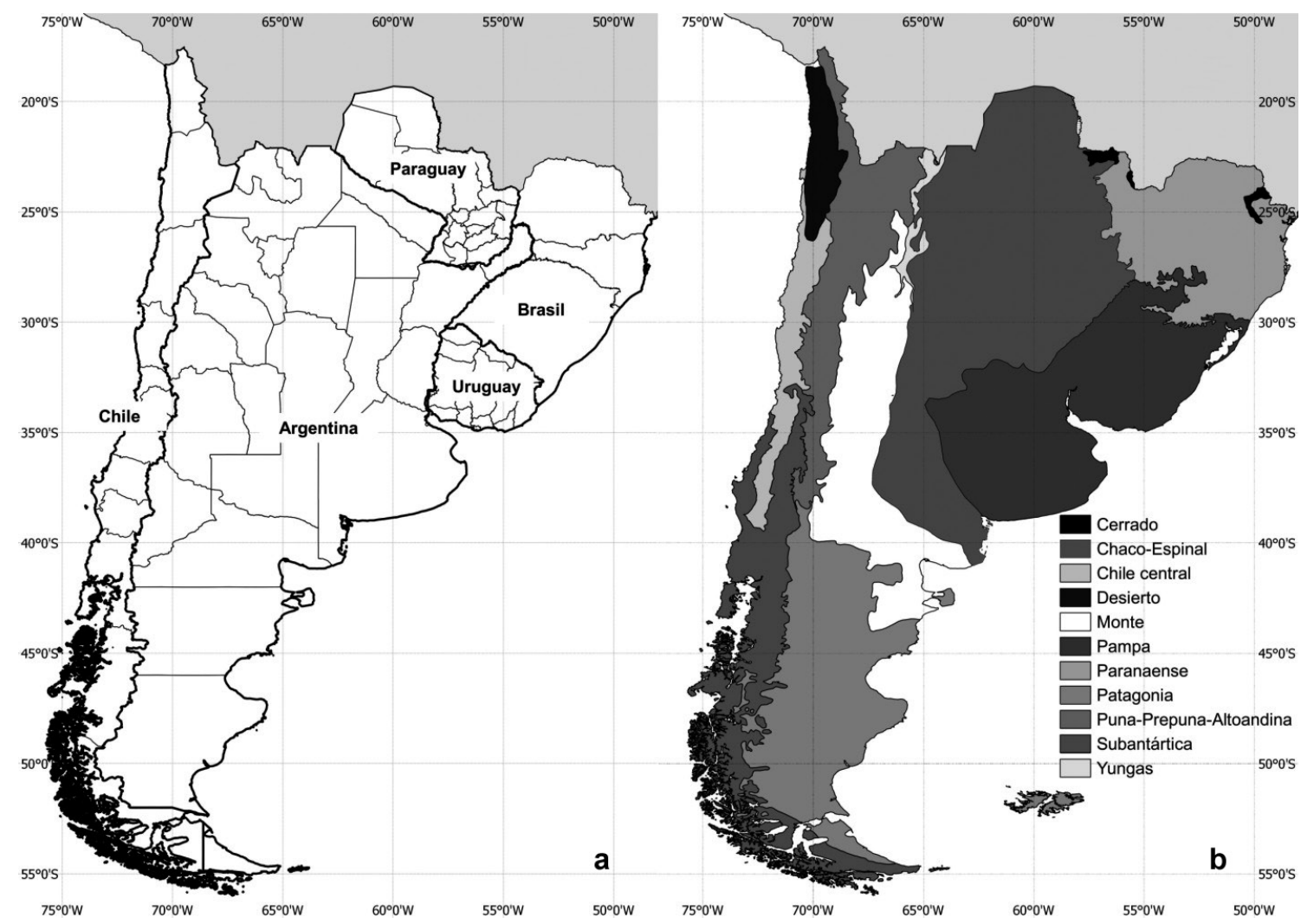

Figura 1 - Área de estudio - a. división política del área de estudio; se representan unidas algunas subdivisiones políticas de Chile, Paraguay y Uruguay para representar gráficamente unidades de tamaños similares; b. división biogeográfica del área de estudio; se representan unidas las provincias altoandina, prepuneña y puneña (sensu Cabrera \& Willink 1973).

Figure 1 - Study area - a. political division of the study area; we join some political subdivisions of Chile, Paraguay and Uruguay to plot units of similar sizes; b. biogeographic division of the study area; we join altoandina, prepuna and puna provinces for clarity (sensu Cabrera \& Willink, 1973).

Steud. (Chile), Nicoraepoa Soreng \& L.J. Gillespie (Argentina y Chile), Podophorus Phil. (Chile) y Relchela Steud. (Argentina y Chile) y Triticeae: Eremium Seberg \& Linde-Laursen (Argentina). Los géneros con mayor número de especies endémicas son Nassella (74), Poa (44), Festuca (30), Paspalum (27), Pappostipa (26), Melica L. (25) y Aristida L. (18) (Apéndice Tab. 3).

Diversidad por ecorregiones: A nivel de géneros, la provincia paranaense es la que tiene mayor diversidad con 97 géneros, seguida por la pampeana (84) y la chaqueña (83) (Apéndice Tab. 5; Fig. 2a). La mayoría de los géneros están compartidos por más de una provincia $\mathrm{y}$, por ejemplo, sólo 14 de los 97 presentes en la provincia paranaense o 7 de los 30 presentes en el cerrado son exclusivos; el resto de las provincias tienen 5 o menos géneros exclusivos (Apéndice Tab. 5; Fig. 2b). A nivel de especies, la provincia paranaense es la que tiene mayor número de especies (428), seguida por la pampeana, chaqueña y altoandina con 394, 307 y 260 respectivamente (Apéndice Tab. 5; Fig. 2c). De las especies de cada región, una fracción son exclusivas; así, las provincias con mayor número de especies exclusivas son paranaense (164, $38 \%)$, altoandina $(134,51 \%)$ y pampeana $(114$, 29\%) (Apéndice Tab. 5, 6; Fig. 2d). Sin embargo, la mayoría de las especies están compartidas por dos o más regiones. El mayor número de especies compartidas se da entre las provincias chaqueña y pampeana (181), paranaense y chaqueña (147) y paranaense y pampeana (138) (Apéndice Tab. 6).

Cada subfamilia tiene una distribución característica en el área de estudio. Así, entre las subfamilias más diversas, las Pooideas tienen el mayor número de especies en las provincias altoandina, pampeana y patagónica, las Panicoideas están mayoritariamente representadas en las 

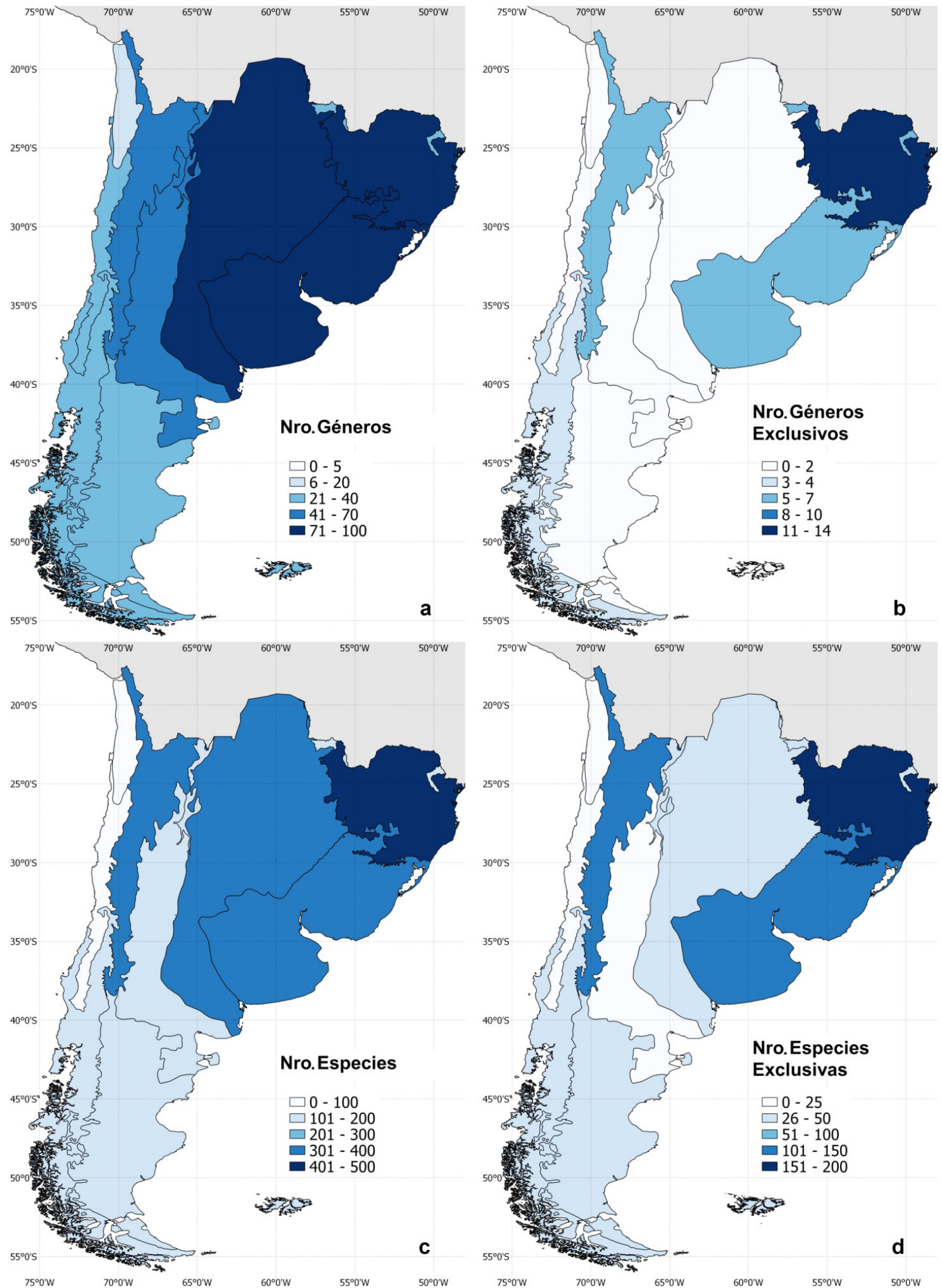

b
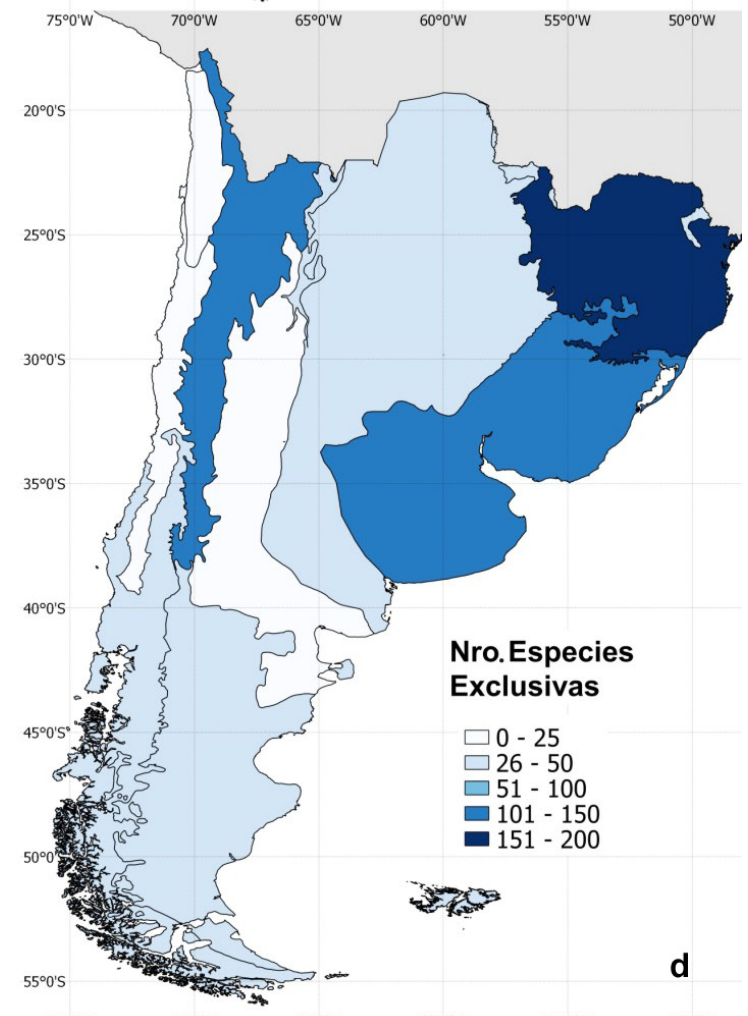

Figura 2 - Distribución del número de géneros y de especies totales (2a-c) y exclusivos (2b-d) en las provincias biogeográficas del sur de América del Sur.

Figure 2-Distribution of the total (2a-c) and exclusive (2b-d) number of genera and species in the biogeographic provinces of southern South America. 
provincias paranaense, chaqueña y pampeana, mientras que las Chloridoideas dominan en las provincias chaqueña, pampeana y monte (Apéndice Tab. 7).

Taxones Kranz y no Kranz: En el Cono sur se observa un patrón general de disminución de la proporción de especies $\mathrm{C}_{4}$ con la latitud considerando las especies nativas de todas las subfamilias (Fig. 4a). Este patrón de variación latitudinal es conocido y se ha reportado para otras regiones (Sage et al., 1999). Sólo la subfamilia Panicoideae incluye especies $\mathrm{C}_{3} \mathrm{y} \mathrm{C}_{4}$, el $11.3 \%$ de las especies presentes en el área son $\mathrm{C}_{3} \mathrm{o}$ intermedias (49 y 4 spp., todas nativas) y el $88.7 \%$ de las especies son $\mathrm{C}_{4}$ (416 spp., de las cuales 54 son introducidas (Apéndice Tab. 8)). De las 7 tribus de la subfamilia Panicoideae, las Paniceae y Paspaleae son las únicas que tienen especies $\mathrm{C}_{3} \mathrm{y} \mathrm{C}_{4} \mathrm{y}$ en ambas la proporción de cada tipo fotosintético es similar (Apéndice Tab. 8). En relación con las diferentes regiones biogeográficas, se puede observar que las Anomochlooideae y Bambusoideae, no Kranz, se hallan en la provincia paranaense y en las yungas principalmente, con una importante disyunción en la tribu Bambuseae (Bambusoideae) en los bosques templados en el sur. Las subfamilias $\mathrm{C}_{3}$ Arundinoideae, Danthonioideae y Pooideae, son dominantes al aumentar la latitud y la altitud, es decir predominan en la región andina, la Patagonia, el sur de la región pampeana y también en los bosques templados. Por el contrario, la subfamilia Ehrhartoideae, también $\mathrm{C}_{3}$, se halla, en su mayor parte, en lugares húmedos y subtropicales a templados de las provincias paranaense y chaqueña. Las subfamilias Aristidoideae y Chloridoideae, ambas $\mathrm{C}_{4}$, son abundantes en el sur de la provincia paranaense (en el distrito de los campos), en la provincia chaqueña y en el norte de la provincia pampeana, y el número de especies es reducido al sur de esta región y en la Patagonia. Finalmente, los representantes $\mathrm{C}_{3}$ de la subfamilia Panicoideae usualmente se hallan en hábitats húmedos y cálidos de la provincia paranaense (su distribución se extiende hacia el sur normalmente a los largo de cursos de agua), mientras que $\operatorname{los} \mathrm{C}_{4}$ se hallan típicamente en ambientes abiertos del sur de la provincia paranaense, en la provincia chaqueña y en el norte de la provincia pampeana; si bien el número de especies $\mathrm{C}_{4}$ disminuye también con la altitud, especies de Paspalum y Cenchrus pueden llegar hasta los $4.200 \mathrm{~m}$ de elevación.

Entre las especies introducidas, el $60 \%$ son especies $\mathrm{C}_{3}$ principalmente de la subfamilia
Pooideae, mientras que las especies introducidas $\mathrm{C}_{4}$ pertenecen a las subfamilias Chloridoideae y Panicoideae (Apéndice Tab. 9).

Anomochlooideae. Representa uno de los linajes basales de la familia (GPWG II, 2012). La integran gramíneas herbáceas características del interior de bosques tropicales (mata atlántica y provincia paranaense en el área del Cono Sur), las que poseen hojas dísticas o espiraladas, pseudopecioladas y con un pulvínulo conspicuo, mientras que las lodículas están ausentes y el número básico de cromosomas de la subfamilia es $x=9$ u 11. En el Cono Sur la subfamilia está representada por un único género, Streptochaeta Schrad. ex Nees, con una única especie, S. spicata Schrad. ex Nees, frecuente en Brasil y Paraguay y ocasionalmente hallada en el noreste argentino (Misiones) (Fig. 4a).

Aristidoideae. Son gramíneas frecuentes en pastizales y sabanas templados. Son plantas anuales o perennes, cespitosas, con una inflorescencia contraída o laxa con espiguillas unifloras, perfectas, la lemma endurecida y con 3 aristas y cariopsis con hilo linear; el número básico de cromosomas es $x=11,12$. Cabe destacar que si bien Aristida se ha clasificado, hasta el momento, como Kranz del subtipo NADP-me (excepcionalmente con dos vainas alrededor de los haces vasculares), recientemente Cerros-Tlatilpa \& Columbus (2009) citaron el hallazgo de una especie no Kranz en el género. Comprende en el área un único género, Aristida, con 44 especies, de las que la mayoría crece en el NE de la Argentina, Uruguay, Brasil y Paraguay (Fig. 4b). Dieciocho de las 44 especies de Aristida son endémicas del Cono Sur, y crecen la mayoría en las provincias chaqueña y pampeana.

Arundinoideae. Esta subfamilia se distingue por incluir taxones con plantas robustas, espiguillas lateralmente comprimidas, desarticulando por encima de las glumas multifloras, con cariopsis con hilo punctiforme a oval, endosperma duro; el epiblasto está ausente y el mesocótilo posee el entrenudo alargado, mientras que el surco entre la base del escutelo y la coleorriza está presente y los márgenes de las hojas embrionales no están superpuestas. La subfamilia incluye plantas no-Kranz, XyMS+ y un número básico de cromosomas $x=12$. Posee sólo dos géneros en la región: uno introducido en América, Arundo L. y otro cosmopolita, Phragmites Adans., ambos abundantes desde el nivel del mar hasta aproximadamente $2500 \mathrm{~m}$ s.m. Phragmites crece 
hasta la Patagonia en la Argentina (Fig. 4c), y es poco frecuente, o está ausente, en el este del Cono Sur en las provincias chaqueña y paranaense.

Bambusoideae. La subfamilia incluye a los tradicionalmente llamados "bambúes", tanto a los herbáceos como a los "leñosos", particularmente diversos y abundantes en selvas tropicales de todo el mundo. Se distingue por incluir plantas perennes, con inflorescencias en panojas abiertas a racemosas o espiciformes, llevando espiguillas o pseudoespiguillas, siendo el fruto un aquenio o una cariopsis con hilo lineal, el endosperma es duro; epiblasto presente; mesocótilo con entrenudo inconspicuo, con el surco entre la base del escutelo y la coleorriza presente y los márgenes de las hojas embrionales superpuestos; las plantas son no-Kranz, XyMS+, siendo frecuentes en el mesófilo las células fusoides y las células de paredes lobuladas ("arm cells"); el número básico de cromosomas es $x=7$. Dentro del área de estudio se encuentran dos tribus, Bambuseae que incluye a los bambúes "leñosos" y la de mayor número de especies, y la tribu Olyreae que comprende a todos los bambúes herbáceos (Fig. 4d). La tribu Bambuseae posee en el Cono Sur 8 géneros, de los cuales Apoclada McClure, Aulonemia Goudot y Cambajuva P.L. Viana, Filg. \& L.G. Clark son exclusivos de Brasil, Rhipidocladum McClure lo es del noroeste de la Argentina (llega a las Yungas desde Bolivia), mientras que Chusquea Kunth, Colanthelia McClure ex E.W. Sm. y Merostachys Spreng. crecen en el sur de Brasil, Paraguay y la Argentina. Chusquea es el único representante de la subfamilia en Chile mientras que en Uruguay sólo crece el género Guadua Kunth, género que también está presente en la Argentina, Brasil y Paraguay. Es interesante la disyunción del género Chusquea, con especies que crecen en el sur de Brasil, Paraguay y noreste de la Argentina (e.g. $C$. juergensii Hack., C. ramosissima Pilg.), otras en el noroeste de Argentina (e.g. C. deficiens Parodi) y otro grupo exclusivo de los bosques subantárticos de Argentina y Chile (e.g., C. culeou E. Desv., $C$. quila Kunth y $C$. valdiviensis E. Desv.). Chusquea fernandeziana Phil. es una de las 5 gramíneas endémicas de las islas de Juan Fernández, Chile. En lo que respecta a los bambúes herbáceos, la tribu Olyreae comprende 4 géneros que crecen en selvas del norte de la Argentina, Brasil y Paraguay, en la provincia biogeográfica paranaense: Lithachne $\mathrm{P}$. Beauv., Parodiolyra Soderstr. \& Zuloaga, Reitzia Swallen (exclusivo de Brasil) y Olyra L., los 3 primeros con una especie y Olyra con un total de 5 especies. Es claro que la distribución de la subfamilia está asociada a la presencia de bosques subtropicales en el Cono Sur (además de la señalada disyunción con los bosques templados en el género Chusquea), tanto en la provincia de las yungas (entre Bolivia y Argentina), como en la provincia paranaense en el este de Paraguay, sur de Brasil y norte de la Argentina.

Chloridoideae. Es dominante en ambientes áridos o semiáridos y se caracteriza por incluir taxones con espiguillas perfectas, estaminadas y pistiladas, lateralmente comprimidas, unifloras o multifloras, cariopsis con hilo oval o punctiforme; epiblasto usualmente presente, mesocótilo con entrenudo manifiesto, alargado; surco entre la base del escutelo y la coleorriza presente y márgenes de las hojas embrionales adnatos, no superpuestos; son plantas Kranz, con número básico de cromosomas $x=(7,8-) 9,10(-12)$. Incluye en el área 3 tribus, Cynodonteae, Eragrostideae y Zoysieae, con un total de 31 géneros y 196 especies (Fig. 4e). La tribu Cynodonteae es la mejor representada con 26 géneros y un total de 126 especies, 29 de las cuales son endémicas; Chloris Sw. es el género más diverso con un total de $18 \mathrm{spp}$. El género Neobouteloua es endémico, con dos especies que crecen en regiones áridas y semiáridas de la Argentina mientras que el género Dactyloctenium es introducido. Algunos géneros tienen una interesante distribución disyunta anfitropical, como Blepharidachne Hack., Distichlis Raf., Erioneuron Nash, Munroa Torr., Pappophorum Schreb., Scleropogon Phil. y Willkommia Hack., con algunas especies en el sur de los Estados Unidos de América y México y otras en regiones secas de América del Sur (Peterson et al. 2007). La tribu Eragrostideae comprende a los géneros Cottea Kunth, Enneapogon Desv. ex P. Beauv. y Eragrostis, los dos primeros géneros son también disyuntos (con representantes también en América del Norte), mientras que Eragrostis, el género con mayor número de especies (46), se distribuye por toda América y está presente en África y Australia. Finalmente, la tribu Zoysieae incluye a los géneros Spartina, con 5 spp. características de suelos salobres, y a Sporobolus R. Br. con 17 spp. En líneas generales, las Chloridoideae son más diversas y abundantes en climas áridos y cálidos (Hartley \& Slater 1960). Peterson et al. (2007) señalan un patrón de distribución, de la subfamilia, en América del Sur, el que incluye 
básicamente áreas chaqueñas, y de pampas y sabanas, de Brasil, Paraguay, el este de Bolivia y noroeste y centro de la Argentina; estos autores mencionan un importante número de especies endémicas dentro de este patrón. El presente análisis concuerda con el criterio de Peterson et al. (2007), quienes hallaron que la subfamilia domina en las provincias chaqueña, pampeana y áreas con campos de la provincia paranaense, es decir lugares abiertos y mayormente secos.

Danthonioideae. Se caracteriza por incluir taxones con inflorescencias multifloras, que llevan espiguillas perfectas o diclinas, desarticulando por encima de las glumas y entre los antecios, cariopsis con hilo punctiforme a linear, endosperma duro, epiblasto ausente, mesocótilo con entrenudo manifiesto y surco entre la base del escutelo y la coleorriza presente; las plantas son no-Kranz, XyMS+ y el número básico de cromosomas es variable, $x=6,7,9$. Esta subfamilia incluye la tribu Danthonieae con 4 géneros y 28 especies (Fig. 4f). Cortaderia es un género con cerca de 24 especies distribuidas en América del Sur, Nueva Zelanda y Nueva Guinea; en el Cono Sur el género crece desde el norte de Argentina y Chile hasta Patagonia y los bosques subantárticos en el sur, aunque está ausente en Paraguay y en toda la región chaqueña. El género cosmopolita Danthonia DC. comprende 12 taxones, uno introducido, distribuidos desde la Puna y la provincia paranaense hasta los bosques subantárticos; Rytidosperma Steud., género presente también en África, Asia, Australia y Nueva Zelanda, con 6 especies en la Patagonia y de bosques subantárticos de la Argentina y Chile; Schismus P. Beauv. es un género introducido con dos especies creciendo en el sur de la Argentina.

Ehrhartoideae. Son plantas anuales o perennes, con espiguillas bisexuales o unisexuales, desarticulando por debajo de las glumas, usualmente con 1 antecio perfecto o unisexual, el fruto una cariopsis o aquenio, embrión con epiblasto usualmente presente, mesocótilo con entrenudo ausente o poco manifiesto, surco entre la base del escutelo y la coleorriza usualmente presente y márgenes de las hojas embrionales superpuestos; las plantas son no-Kranz, XyMS+ y el número básico de cromosomas $x=12$ $(10,15,17)$. Esta subfamilia incluye la tribu introducida Ehrharteae con una especie del género Ehrharta P. Beauv., y la tribu Oryzeae, con 5 géneros y 17 especies (Fig. 4g). Ehrharta es un género introducido que sólo se halla en dunas costeras en la Argentina; Leersia Sw., dentro de las Oryzeae, incluye 3 especies, típicas de zonas bajas e inundables de Argentina, Brasil, Paraguay y Uruguay (el género no está presente en Chile); una distribución similar se observa en los géneros Luziola Juss. (7 spp.), Oryza (3 spp., una introducida), Rhynchoryza Baill. (1 sp.) y Zizaniopsis Döll \& Asch. (2 spp.), típicas de las regiones Chaqueña, Pampeana y Paranaense en el este del Cono Sur.

Panicoideae. Esta subfamilia es la segunda en cantidad de especies en el Cono Sur e incluye 469 especies y 64 géneros repartidos en 7 tribus: Andropogoneae, Arundinelleae, Gynerieae, Paniceae, Paspaleae, Tristachyideae y Zeugiteae. Se distribuyen principalmente en áreas de cerrados, en pampas y en la provincia chaqueña (Fig. 4h), o sea en áreas subtropicales y templado cálidas, disminuyendo su representación hacia la región Andina y la Patagonia (en regiones templadas a frías). Las tribus más diversas son las Andropogoneae (26 géneros, $101 \mathrm{spp}$.), las Paniceae (22 géneros, 190 spp.) y las Paspaleae (20 géneros, $171 \mathrm{spp}$.). Las Panicoideae fueron caracterizadas (GPWG I, 2001) por incluir plantas básicamente herbáceas, dominantes en regiones tropicales y subtropicales, con espiguillas usualmente comprimidas dorsiventralmente, desarticulando por debajo de las glumas, con dos lodículas, dos estigmas, endosperma endurecido, con granos de almidón simples, menos frecuentemente compuestos, embrión usualmente sin epiblasto y con surco entre la base del escutelo y la coleorriza presente. El número básico de cromosomas varía en la subfamilia y es $x=5-9,10(12,14)$. Es la única subfamilia, en el Cono Sur, que presenta los tipos fotosintéticos $\mathrm{C}_{3} \mathrm{y} \mathrm{C}_{4}$, este último incluyendo los subtipos PEP$\mathrm{CK}, \mathrm{NAD}-\mathrm{ME}$ and NADP-ME; existen también

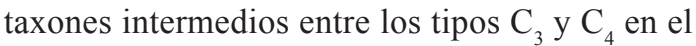
género Steinchisma Raf. El síndrome Kranz es constante en varias tribus, como Andropogoneae, Arundinelleae y Tristachyideae, y variable en otras, como Paniceae y Paspaleae (Morrone et al. 2012), lo que indica que el carácter ha evolucionado varias veces en las Panicoideae (Giussani et al. 2001).

Andropogoneae. Incluye taxones Kranz, XyMS-, con espiguillas sésiles bisexuales y espiguillas pediceladas estaminadas o neutras; el número básico de cromosomas es usualmente $x=$ 
5, ocasionalmente 9 (Clayton \& Renvoize 1986). Comprende 26 géneros y 101 especies (Andropogon L., Bothriochloa Kuntze y Schizachyrium Nees son los géneros con más especies con 17, 15 y 15, respectivamente). Cabe destacar que 9 de estos géneros son introducidos: Chrysopogon, Coix L., Cymbopogon Spreng., Dichanthium Willemet, Hemarthria R. Br., Miscanthus, Rottboellia L.f., Sorghum Moench. y Themeda Forssk. Agenium Nees es el único restringido a América del Sur.

Arundinelleae. Es cosmopolita y la mayoría de los géneros que la integran crecen en trópicos del viejo mundo. En el Cono Sur sólo se encuentra, en campos o sabanas de las regiones chaqueña y paranaense, Arundinella Raddi, Kranz del subtipo XyMS- y 2 spp.

Gynerieae. Tribu monotípica con un género neotropical, Gynerium Willd. ex P. Beauv., ampliamente distribuido, en márgenes de ríos o en áreas inundadas, desde México y el Caribe hasta la Argentina; se distingue por poseer hojas dísticas, rígidas, dioecia e inflorescencias pistiladas plumosas. El género es no-Kranz y posee un número básico de cromosomas de $x=11$.

Paniceae. Esta tribu ha sido delimitada por diferentes autores (Pilger 1940; Hsu 1965; Butzin 1970; Brown 1977; Clayton \& Renvoize 1986) básicamente por incluir espiguillas dispuestas en inflorescencias laxas, abiertas, espiguillas usualmente comprimidas dorsiventralmente, bifloras, la flor inferior estaminada o ausente, la superior perfecta, el antecio superior endurecido. El número básico de cromosomas varía entre $x=9$ y $x=10$. En esta tribu, al igual que en Paspaleae, se han descripto dos tipos anatómicos y fotosintéticos. Recientemente, Morrone et al. (2012) segregan, sobre la base de caracteres moleculares, a la tribu Paspaleae, la que incluye géneros con un número básico de cromosomas $x=10$, siendo los caracteres exomorfológicos similares a los descriptos para las Paniceae. Es así que la tribu Paniceae quedó restringida (Morrone et al. 2012) a géneros y especies anuales y perennes con inflorescencias laxas, abiertas, con un número básico de cromosomas $x=9$; incluye taxones Kranz y no Kranz. Dado que se ha comprobado que el género tipo de la tribu, Panicum, no es monofilético (Giussani et al. 2001; Aliscioni et al. 2003), en el presente tratamiento se considera al mismo restringido a especies con inflorescencias abiertas, sin espiguillas dispuestas en racimos unilaterales, con antecio superior endurecido con un tipo fotosintético Kranz, del subtipo NAD-ME $\mathrm{y}$ un número básico de cromosomas $x=9$. En consecuencia, diversas especies del género han sido transferidas a otros géneros de la tribu Paniceae y de las Paspaleae. La tribu Paniceae posee en el Cono Sur 21 géneros, de los cuales Acroceras Stapf, Cenchrus, Digitaria Haller, Echinochloa P. Beauv., Eriochloa Kunth, Oplismenus P. Beauv., Panicum, Pseudechinolaena Stapf, Sacciolepis Nash, Setaria P. Beauv., Trichanthecium Zuloaga \& Morrone y Urochloa son cosmopolitas, Dichanthelium Gould, Lasiacis (Griseb.) Hitchc., Morronea Zuloaga \& Scataglini, Parodiophyllochloa Zuloaga \& Morrone y Stenotaphrum son americanos, Megathyrsus (Pilg.) B.K. Simon \& S.W.L. Jacobs, Melinis P. Beauv. y Moorochloa Veldkamp son introducidos y Louisiella C.B. Hubbard \& J. Leónard es un anfitropical disyunto, con una especie ampliamente distribuida en América, sumergida en cursos de agua (en el Cono Sur en las regiones chaqueña, paranaense y pampeana) y otra en África. De estos géneros, 9 son noKranz (Acroceras, Dichanthelium, Lasiacis, Morronea, Oplismenus, Parodiophyllochloa, Pseudechinolaena, Sacciolepis, Trichanthecium), 11 son Kranz (Cenchrus, Digitaria, Echinochloa, Eriochloa, Louisiella, Megathyrsus, Melinis, Moorochloa, Setaria, Stenotaphrum y Urochloa). Panicum aún incluye, con especies "incertae sedis", taxones Kranz y no-Kranz.

Paspaleae. La tribu comprende un total de 20 géneros, la mayoría americanos; sólo Axonopus P. Beauv., Hymenachne P. Beauv., Ichnanthus P. Beauv. y Paspalum poseen un número restringido de especies en el viejo mundo. La mayoría de los géneros posee una distribución mayoritariamente en áreas tropicales y subtropicales, si bien un número importante de especies de Paspalum crecen en regiones andinas, llegando hasta los 4.300 $\mathrm{m}$ s.m. Oplismenopsis y Osvaldoa son géneros endémicos del área, creciendo ambos en ambientes húmedos, orillas de ríos y arroyos en Argentina, Brasil y Uruguay (Osvaldoa) y Argentina y Uruguay (Oplismenopsis). Esta es la otra tribu con los dos tipos fotosintéticos, con el 85\% de las especies Kranz; los géneros no-Kranz son Canastra Morrone, Zuloaga, Davidse \& Filg., Echinolaena Desv., Homolepis Chase, Hymenachne, Ichnanthus, Ocellochloa Zuloaga \& Morrone, Otachyrium Nees, Rugoloa Zuloaga y Stephostachys Zuloaga $\&$ Morrone, todos creciendo principalmente en la provincia paranaense, mientras que Steinchisma, 
género que llega a áreas húmedas de la provincia pampeana, incluye especies intermedias $\mathrm{C}_{3} / \mathrm{C}_{4}$.

Tristachyideae. Está representada por 3 géneros con una especie cada uno: Loudetia Hochst. ex Steud., Loudetiopsis Conert y Tristachya Nees, presentes en cerrados y campos de la provincia paranaense de Brasil y Paraguay. Se caracteriza por espiguillas en tríadas, con la lemma superior aristada, la arista a menudo decidua. Los géneros tienen un número básico de cromosomas $x=6,7$, 10, 12, and 14 y son Kranz, XyMS-.

Zeugiteae. Comprende un único género no Kranz, Orthoclada P. Beauv., con una especie de distribución tropical en América y una especie en África; el número básico de cromosomas es $x=12$.

Pharoideae. Representa, al igual que las Anomochlooideae, una de las subfamilias basales de las Poaceae e incluye un único género, Pharus P. Browne (2 ssp.), caracterizado por poseer hojas pseudopecioladas y giradas $180^{\circ}$, de modo tal que la cara abaxial está dispuesta hacia arriba en las plantas (a su vez las hojas muestran una venación oblicua y no son paralelinervias); las plantas son monoicas y tanto las espiguillas estaminadas como pistiladas poseen dos glumas y un antecio; en las espiguillas pistiladas el antecio está endurecido y cubierto de pelos en forma de gancho, los que sirven para la dispersión de las plantas por los animales. El número básico de cromosomas es $x=12$. Pharus es una gramínea herbácea que crece en el interior de selvas y bosques tropicales y subtropicales, siendo común en áreas de la provincia paranaense del norte de la Argentina, Brasil, Paraguay y Uruguay (Fig. 4i).

Pooideae. Es la subfamilia más diversa con 691 spp. pertenecientes a 74 géneros organizados en 6 tribus (Fig. 4j). Las tribus con mayor número de especies son las Poeae y Stipeae con 355 y 202, respectivamente. Esta subfamilia tiene el mayor porcentaje de especies endémicas en el área de estudio (54\%, 372 spp.), así como también el mayor porcentaje de especies introducidas (16\%, 109 spp.). Su distribución en la región está claramente definida por la presencia de la cordillera de los Andes y por el aumento de latitud. Es así que la subfamilia es sumamente diversa y rica en todo el norte de Chile y noroeste de la Argentina, en la región andina por encima de los 1500-2000 $\mathrm{m}$, siendo dominantes hacia el sur a lo largo de los Andes, extendiendo luego este predominio al centro y sur de la provincia pampeana y a toda la Patagonia; también es significativa su presencia en las islas chilenas analizadas. La subfamilia comprende plantas anuales o perennes con láminas lineares, espiguillas 1-2 a multifloras, usualmente desarticulando por encima de las glumas, cariopsis con embrión pequeño, usualmente con epiblasto, mesocótilo con entrenudo no manifiesto, surco entre la base del escutelo y la coleorriza ausente; el hilo varía de punctiforme a linear, llegando a ser tan largo como la cariopsis; son plantas no Kranz y el número básico de cromosomas es de $x=5,7,8$, $9,10,11$ y 12 . Incluye en el Cono Sur un total de 6 tribus, siendo las Poeae las mejor representadas.

Brachypodieae. Es una tribu monotípica con 2 especies introducidas en la Argentina en la región pampeana.

Bromeae. Esta tribu tiene un género en el área, Bromus, con un total de 33 spp., de las cuales 10 son endémicas y 16 son introducidas; sus especies son, por lo general, importantes en pastizales de regiones templadas y montañosas hasta $4500 \mathrm{~m}$ s.m.

Meliceae. Son plantas perennes, con vainas cerradas, la inflorescencia es una panoja laxa a contraída, llevando espiguillas multifloras, con los antecios basales perfectos y los distales reducidos; el número básico de cromosomas es $x=9,10$. Tiene 2 géneros en el área, Glyceria R. Br., propio de ambientes húmedos, palustres o acuáticos, con 3 especies, una endémica en el noroeste argentino (provincia de Salta), 1 nativa y otra introducida en la región central de Chile. Por otra parte, Melica, género con aproximadamente 80 especies de regiones templadas de ambos hemisferios, incluye 25 spp. endémicas y 5 nativas, siendo muchas de ellas importantes elementos en pastizales pampeanos, llegando $M$. chilensis J. Presl hasta los $3700 \mathrm{~m}$ s.m. Melica sarmentosa Nees es una planta trepadora, que llega a los $3 \mathrm{~m}$ de altura, propia de márgenes de bosques y selvas en ambientes húmedos.

Poeae. Es la tribu con mayor riqueza y variedad dentro de la subfamilia; comprende plantas anuales o perennes, con lígulas membranáceas a hialinas, raro ciliadas, espiguillas desarticulando por encima de las glumas y entre los antecios, uni a multifloras, cariopsis con hilo punctiforme a linear y número básico de cromosomas $x=7$. Tiene 51 géneros, entre los que hay 5 endémicos, Leptophyllochloa, Megalachne, Podophorus y Relchela, el primero y el último en ambientes húmedos, de los bosques andino-patagónicos, de la Argentina y Chile, Nicoraepoa, con 7 spp. propias 
de regiones andinas y patagónicas de la Argentina y Chile, mientras que Megalachne y Podophorus son endémicos de las islas de Juan Fernández, Chile. Los géneros mejor representados, distribuidos a lo largo de la cordillera de los Andes y extendidos en la Patagonia y provincia pampeana, son Poa, con 77 spp., Festuca, con 54, Deyeuxia Clarion ex P. Beauv. con 45 y Agrostis L. con 32.Las Poeae tienen un total de 19 géneros introducidos, los que crecen principalmente en las regiones patagónica y pampeana; la mayor parte incluye 1 a 2 especies, Avena tiene 6, Lolium 5 y Aira L. 4.

Stipeae. Se caracteriza por incluir plantas perennes, rara vez anuales, con lígulas membranáceas, láminas lineares, espiguillas dispuestas en panojas terminales, unifloras, con raquilla articulada por encima de las glumas, lemma rígida, con los bordes convolutos o involutos sobre la pálea, aristada y cariopsis con hilo linear. Sus especies se encuentran distribuidas en ambos hemisferios, en particular en estepas y praderas y también en regiones montañosas. La actual delimitación la tribu comprende un total de 29 géneros (Romaschenko et al. 2012), de los que en el Cono Sur hay 1 género introducido, Austrostipa S.W.L. Jacobs \& J. Everett, y 9 géneros nativos: Aciachne Benth., con 1 spp. nativa, Amelichloa Arriaga \& Barkworth con 4 spp. (3 endémicas), Anatherostipa (Hack. ex Kuntze) Peñail. con 8 spp. (4 endémicas), Jarava Ruiz \& Pav. con 26 spp. (17 endémicas), Nassella con 97 spp. (75 endémicas), Ortachne Nees ex Steud. con 2 spp. (1 endémica), Pappostipa con 29 spp. (27 endémicas), Piptatherum P. Beauv. con 1 sp. y Piptochaetium J. Presl con 25 spp. (17 endémicas).

Triticeae. Se distingue por incluir especies en las que, usualmente, la inflorescencia es una espiga, con 1 a 3 espiguillas sésiles, desarticulando por encima de las glumas y debajo de los antecios, lateralmente comprimidas, cariopsis con hilo linear y número básico de cromosomas $x=7$. La tribu se distribuye en regiones templadas y frías del Cono Sur; la mayoría de sus taxones crecen en Argentina y Chile, llegando hasta Uruguay. Las Triticeae tienen una distribución preponderante en el hemisferio norte, del que provienen 3 géneros
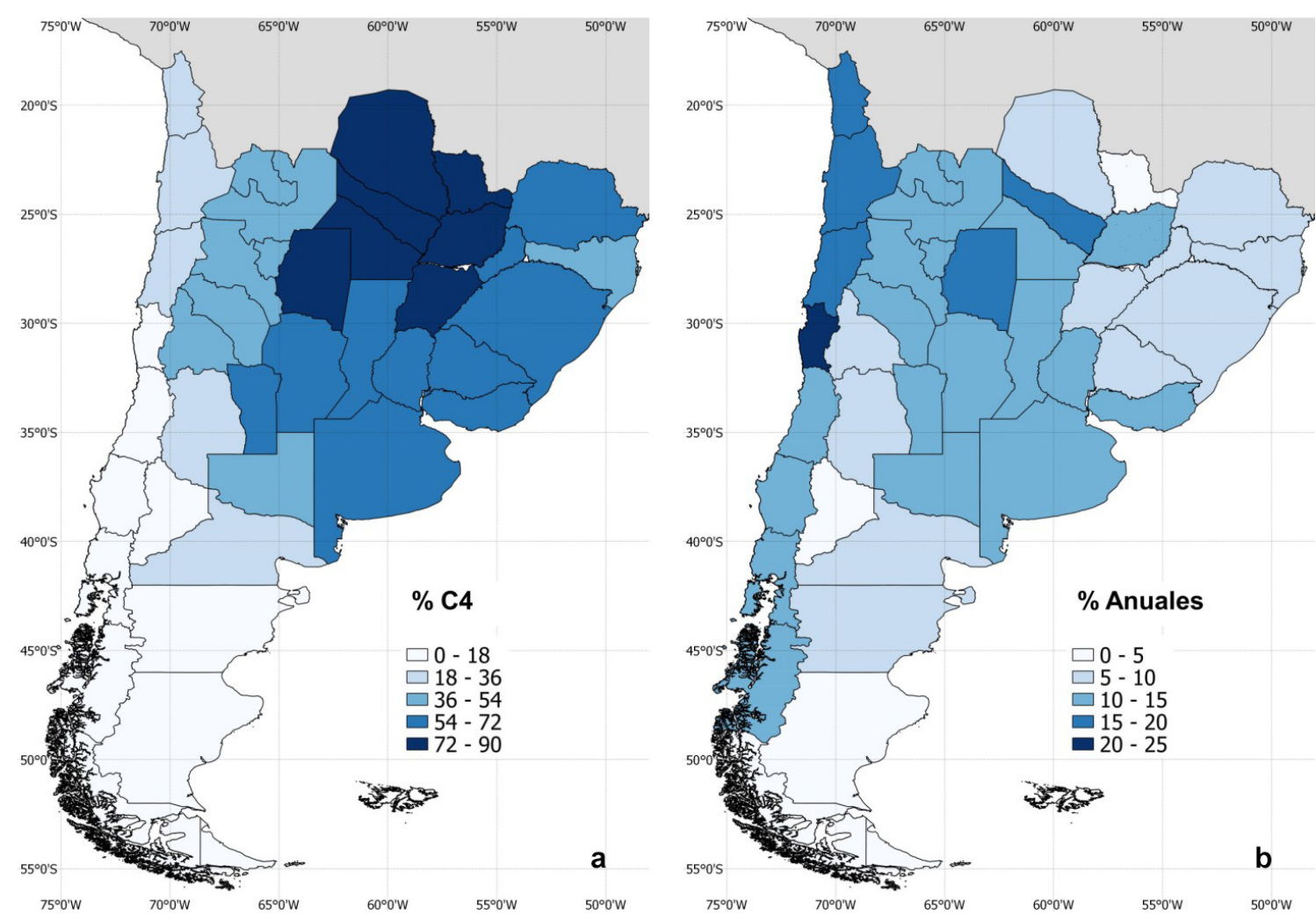

Figura 3 - Distribución del porcentaje de especies nativas de Poaceae $\mathrm{C}_{4}$ (3a) y anuales (3b) en el sur de América del Sur. Figure 3 - Distribution of percentage of native $C_{4}$ (3a) and annual (3b) species of Poaceae in southern South America. 


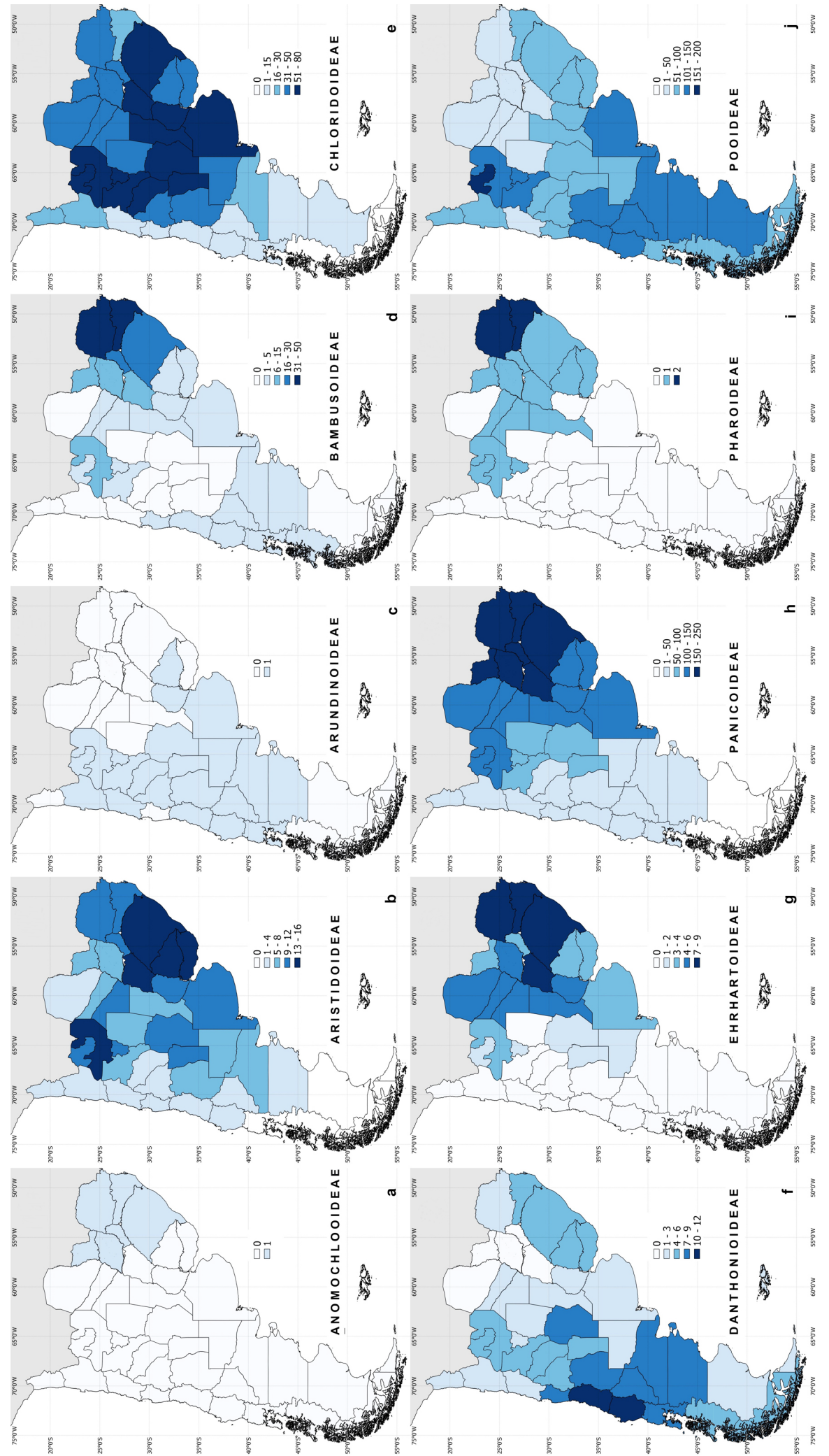

Figura 4-Distribución del número de especies nativas para las 10 subfamilias de Poaceae presentes en el sur de Améroca del Sur. Figure 4 - Distribution of the number of native species for the 10 subfamilies of Poaceae present in southern South America. 
introducidos: Leymus Hochst., Elytrigia Desv. y Thinopyrum Á. Löve; Eremium es endémico en regiones andinas de la Argentina, desde la provincia de La Rioja hasta Santa Cruz (alcanzando los $3400 \mathrm{~m}$ s.m.), mientras que Elymus L. posee 5 spp. endémicas y 2 introducidas y Hordeum cuenta con un total de 21 spp., 12 endémicas, 4 introducidas y 5 nativas.

Disyunciones: La mayoría de las disyunciones presentes en el área involucran a las regiones áridas del sur de Estados Unidos y México (Raven 1963; Burkart 1975). En este grupo de géneros disyuntos se incluyen numerosos ejemplos en las Chloridoideae: Blepharidachne, Cottea, Distichlis, Enneapogon, Erioneuron, Munroa, Pappophorum, Scleropogon, Tridens Roem. \& Schult. y Willkommia, y dos en Panicoideae: Bothriochloa y Digitaria. Otro grupo de géneros disyuntos son los que involucran a regiones frías de América del Norte y del Cono Sur y se da en las Pooideae, en Catabrosa P. Beauv., Phippsia (Trin.) R. Br. y Podagrostis (Griseb.) Scribn. \& Merr.

Ciclo de vida: La mayoría de las especies nativas del cono sur son perennes y solo alrededor del $8 \%$ son anuales (Apéndice Tab. 10). Estas especies anuales se concentran en las subfamilias Chloridoideae, Panicoideae y Pooideae. El porcentaje de especies anuales disminuye con la latitud en el Cono sur (Fig. 3b) y los valores más altos se observan en Chile en la región del desierto de Chile y en Chile central. Entre las especies introducidas aproximadamente la mitad de las especies son anuales, principalmente en Pooideae (Apéndice Tab. 9).

Géneros y especies introducidos: En total hay 45 géneros introducidos (Apéndice Tab. 3), 1 de Arundinoideae (Arundineae), Chloridoideae (Cynodonteae), Danthonioideae (Danthonieae) y Ehrhartoideae (Ehrharteae); 13 de Panicoideae (10 de Andropogoneae y 3 de Paniceae) y 28 de Pooideae (1 de Brachypodieae, 20 de Poeae, 2 de Stipeae y 5 de Triticeae). Las especies suman 66, mientras que las restantes $134 \mathrm{spp}$. introducidas en el Cono sur pertenecen a 36 géneros con especies nativas e introducidas.

\section{Conclusiones}

Este estudio permite concluir que la familia Poaceae comprende el 7,75\% de la flora vascular del Cono Sur e incluye el $4,95 \%$ de las especies endémicas que ocurren en el área. Sólo 9 géneros son endémicos, 6 de ellos son monotípicos y otros 2 contienen sólo 2 especies (Nicoraepoa tiene $7 \mathrm{spp}$.); de ellos, 2 se encuentran en la provincia paranaense (Oplismenopsis y Osvaldoa), 2 son endémicos de las islas Juan Fernández (Megalachne y Podophorus), 4 son propios de la región andino-patagónica (Eremium, Leptophyllochloa, Nicoraepoa y Relchela), mientras que Neobouteloua crece en la provincia del monte. La mayor parte de los géneros ricos en especies endémicas tienen una distribución andino-patagónica. Anivel específico, la mayor diversidad de endémicos se registra en la región andina y en la patagonia, mientras que, en líneas generales, la biodiversidad en la región chaqueña es menor en relación al resto.

En lo que se refiere a la distribución de especies $\mathrm{C}_{3} \mathrm{y} \mathrm{C}_{4}$, estas últimas decrecen en número de este a oeste y de norte a sur.

La introducción de especies ha tenido un mayor impacto en las provincias paranaense y chaqueña que en el resto del Cono Sur. Los géneros introducidos representan aproximadamente el $22 \%$ del total de géneros registrados, mientras que a nivel específico, el $13 \%$ del total son especies introducidas.

En cuanto a la riqueza de subfamilias por región biogeográfica, las Anomochlooideae, Pharoideae y Bambusoideae se hallan principalmente representadas en la provincia paranaense y en las yungas, con distribución disyunta en los bosques subantárticos las Bambusoideae. Las Aristidoideae y Chloridoideae son dominantes en las regiones pertenecientes al Dominio Chaqueño de Cabrera \& Willink (chaco, pampa y monte; Cabrera \& Willink 1973), extendiendo su rango a la provincia paranaense y con menor presencia a lo largo de los Andes. Las Ehrhartoideae y Panicoideae crecen principalmente en la provincia paranaense y chaqueña (disminuyendo la distribución de este a oeste y de norte a sur). Finalmente, las Danthonioideae y Pooideae son más abundantes hacia el sur a los largo de los Andes, y son abundantes también en la patagonia extraandina y la pampa.

\section{Agradecimientos}

Apoyo financiero del CONICET (PID 11220100207) y de la National Geographic Society (8862-10). Agradecemos a L. Aagesen por los valiosos comentarios al manuscrito y a $\mathrm{M}$. Biganzoli por la ayuda con la edición de las figuras.

\section{Referencias}

Aliscioni, S.S.; Giussani, L.M.; Zuloaga, F.O. \& Kellogg, E.A. 2003. A molecular phylogeny of Panicum (Poaceae: Paniceae): tests of monophyly and phylogenetic placement within the Panicoideae. American Journal of Botany 90: 796-821.

Bentham, G. \& Hooker, J.D. 1880. Genera Plantarum. L Reeve, London. Vol. 3, pt. 1, pp. 1074-1215. 
Brown, W.V. 1977. The Kranz syndrome and its subtype in grass systematics. Memoirs of the Torrey Botanical Club 23: 1-97.

Brummitt, R.K. \& Powell, C.E. 1992. Authors of plant names. Royal Botanic Gardens, Surrey. 804p.

Burkart, A. 1975. Evolution of grasses and grasslands in South America. Taxon 24: 53-66.

Butzin, F. 1970. Die systematische Gliederung der Paniceae (The Systematic Arrangement of the Paniceae). Willdenowia 6: 179-192.

Cabrera, A.L. 1976. Regiones fitogeográficas argentinas. Enciclopedia Argentina de Agricultura y Jardinería 2: $1-85$

Cabrera, A.L. \& Willink, A.W. 1973. Biogeografía de América Latina. Serie de Biología OEA. Monografía 13: 1-117.

Cerros-Tlatilpa, R. \& Columbus, T. 2009. C photosynthesis in Aristida longifolia: implications for photosynthetic diversification in Aristidoideae (Poaceae). American Journal of Botany 96: 1379-1387.

Chen, S.L.; Li, D.Z.; Zhu, G.; Wu, Z.L.; Lu, S.L.; Liu, L.; Wang, Z.P.; Sun, B.S.; Zhu, Z.; Xia, N.; Jia, L.; Guo, Z.; Chen, W.; Chen, X.; Yang, G.; Phillips, S.M.; Stapleto, C.; Soreng, R.J.; Aiken, S.G.; Tzvelev, N.N.; Peterson, P.M.; Renvoize, S.A.; Olonova, M.V. \& Ammann, K.H. 2006. Poaceae. In: Wu, Z.Y.; Raven, P.H. \& Hong, D.Y. (eds.). Flora of China. Vol. 22 (Poaceae). Science Press and Missouri Botanical Garden Press, Beijing and St. Louis. 752p.

Chiapella, J. \& Zuloaga, F.O. 2010. A revision of Deschampsia, Avenella, and Vahlodea (Poaceae, Poeae, Airinae) in South America. Annals of the Missouri Botanical Garden 97: 141-162.

Clayton, W.D. \& Renvoize, S.A. 1986. Genera Graminum. Grasses of the World. Kew Bulletin additional series 13: 1-389.

Giussani, L.M.; Cota-Sánchez, J.H.; Zuloaga, F.O. \& Kellogg, E.A. 2001. A molecular phylogeny of the grass subfamily Panicoideae (Poaceae) shows multiple origins of $\mathrm{C}_{4}$ photosynthesis. American Journal of Botany 88: 1993-2012.

GPWG I - Grass Phylogeny Working Group. 2001. Phylogeny and subfamilial classification of the grasses (Poaceae). Annals of the Missouri Botanical Garden 88: 373-457.

GPWG II - Grass Phylogeny Working Group. 2012. New grass phylogeny resolves deep evolutionary relationships and discovers $\mathrm{C}_{4}$ origins. New Phytologist 193: 304-312.

Hartley, W. \& Slater, C. 1960. Studies in the origin, evolution and distribution of the Gramineae. V. The subfamily Festucoideae. Australian Journal of Botany 21: 201-234.

Hitchcock, A.S. 1951. Manual of the grasses of the United States, $2^{\text {nd }}$. ed. Revised by A. Chase. United States Department of Agriculture Miscelaneous Publications 200: 1-1051.
Hsu, C.C. 1965. The classification of Panicum (Gramineae) and its allies, with special reference to the characters of lodicule, style-base and lemma. Journal of the Faculty of Science, University Tokyo, section 3 (Botany) 9: 43-150.

Josse, C.; Navarro, G.; Comer, P.; Evans, R.; FaberLangendoen, D.; Fellows, M.; Kittel, G.; Menard, S.; Pyne, M.; Reid, M.; Schulz, K.; Snow, K. \& Teague, J. 2003. Ecological Systems of Latin America and the Caribbean: A Working Classification of Terrestrial Systems. NatureServe, Arlington, VA. 47p.

Linder, H.P.; Baeza, M.; Barker, N.P.; Galley, C.; Humphreys, A.M.; Lloyd, K.M.; Orlouch, D.A.; Pirie, M. D.; Simon, B.K.; Walsh, N. \& Verboom, G.A. 2010. A generic classification of the Danthonioideae (Poaceae). Annals of the Missouri Botanical Garden 97: 306-364.

Morrone, J. J. 2001. Biogeografía de América Latina y el Caribe. Manuales \& Tesis SEA. Vol 3. Zaragoza, España. 148p.

Morrone, O.; Aagesen, L.; Scataglini, M.A.; Salariato, D.L.; Denham, S.S.; Chemisquy, M.A.; Sede, S.M.; Giussani, L.M.; Kellogg, E.A. \& Zuloaga, F.O. 2012. Phylogeny of the Paniceae (Poaceae: Panicoideae): integrating plastid DNA sequences and morphology into a new classification. Cladistics 28: 333-356.

Parodi, L. 1961. La taxonomía de las gramíneas argentinas a la luz de las investigaciones más recientes. Recent Advances in Botany 1: 125-130.

Peterson, P.M. ; Columbus, J.T. \& Pennington, S.J. 2007. Classification and Biogeography of New World Grasses: Chloridoideae. Aliso 23: 580-594.

Peterson, P.M. ; Romaschenko, K. \& Johnson, G. 2010. A classification of the Chloridoideae (Poaceae) based on multi-gener phylogenetic trees. Molecular Phylogenetics and Evolution 55: 580-598.

Pilger, R. 1940. Gramineae. III: Unterfamilie Panicoideae. In: Engler, A. \& Prantl, K. (eds.). Die Natürlichen Pflanzenfamilien, Zweite Auflage. $2^{\text {nd }}$ ed. Vol. $14 \mathrm{e}$. Engelmann, Leipzig. 208p.

Pilger, R. 1956. Gramineae II. In : Engler, A. \& Prantl, K. (eds.). Die Natürlichen Pfanzenfamilien. Vol. 14. Duncker \& Humblot, Berlin. 225p.

Raven, P.H. 1963. Amphitropical Relationships in the Floras of North and South America. The Quarterly Review of Biology 38: 151-177.

Ribichich, A. M. 2002. El modelo clásico de la fitogeografía de Argentina: un análisis crítico. Interciencia 27: 669-675.

Romaschenko, K.; Peterson, P.M.; Soreng, R.; GarcíaJacas, N.; Furtona, O. \& Susanna, A. 2012. Systematics and evolution of the needle grasses (Poaceae: Pooideae: Stipeae) based on analysis of multiple chloroplast loci, ITS, and lema micromophology. Taxon 61: 18-44. 
Sage, R.F.; Wedin, D.A. \& Li, M. 1999. The biogeography of $\mathrm{C}_{4}$ photosynthesis: patterns and controlling factors. In: Sage, R. \& Monson, R.K. (eds.). $\mathrm{C}_{4}$ plant biology. Academic Press, San Diego. Pp.313-373.

Sánchez-Ken, J.G.; Clark, L.G.; Kellogg, E.A. \& Kay, E.E. 2007. Reinstatement and emendation of subfamily Micrairoideae (Poaceae). Systematic Botany 32: 71-80.

Sánchez-Ken, J.G. \& Clark, L.G. 2010. Phylogeny and a new tribal classification of the Panicoideae s.l. (Poaceae) based on plastid and nuclear sequences and structural data. American Journal of Botany 97: 1732-1748.

Soreng, R.J.; Davidse, G.; Peterson, P.M..; Zuloaga, F.O.; Judziewicz, E.J.; Filgueiras, T.S.; Morrone, O. \& Romaschenko,K. 2011 onwards. A worldwide phylogenetic classification of Poaceae (Gramineae). Disponível en $<\mathrm{http}$ ://www.tropicos. org/projectwebportal.aspx?pagename $=$ Classificati onNWG\&projectid=10>. Acceso a 2 Febrero 2012.

Watson, L. \& Dallwitz, M.F. 1992. The grass genera of the world. Cambridge University Press, Cambridge. 1081p. 